\title{
Multivariate statistical technique over QoS variables to analyze video quality metrics on IEEE 802.11ac networks
}

\author{
Miguel García-Pineda, Santiago Felici-Castell, Jaume Segura-García \\ Dpt. Informàtica, Universitat de València \\ Burjassot, España \\ Email: migarpi@uv.es, felici@uv.es, jsegura@uv.es
}

\begin{abstract}
Resumen-We present the results from a measurementbased performance evaluation of wireless networks based on IEEE 802.11ac standard in an indoor environment, with the aim to analyze their performance under high definition streaming video applications. We focus our study on analyzing the highest performance of these standards using off-theshelf equipment as well as the behavior of Quality of Service variables and how they affect to the video quality. Thus, we have analyzed and measured these variables and have applied a multivariate statistical technique, called Factor Analysis, and finally discuss their behavior.
\end{abstract}

Palabras Clave-multivariate statistical technique, factor analysis, video quality metrics, quality of service, quality of experience

\section{INTRODUCTION}

Big companies like Google, Cisco Systems, Apple and Microsoft predict that by 2020, more than $90 \%$ of Internet traffic will be multimedia content (images, 3D images, High Definition (HD) video and audio, etc.) [1]. With these applications, streaming around home as well as mobility has become an issue.

The IEEE 802.11 Working Group has approved several standards to deliver gigabit rates in Wireless Local Area Networks (WLAN), in particular with the IEEE 802.11ac standard, working only in the $5 \mathrm{GHz}$ band. Theoretically, IEEE 802.11ac has expected multi-station WLAN throughput of 1 gigabit per second (Gbps) and single link throughput of 500 megabits per second (Mbps).

Nevertheless, the actual network performance results in real experimental environments are quite different and require a further analysis [2] [3]. Then, it is very important to perform a comprehensive analysis of IEEE 802.11ac network using relatively new off-the-shelf commercial products to validate these specifications, under the scenarios for which they were designed, in particular for video streaming with Full and Ultra HD (or 4K) videos under a subjective video quality point of view or Quality of
Experience (QoE), measured in terms of Mean Opinion Score (MOS).

Thus, in order to detect critical issues in this process, we measure and analyze different variables in the streaming process related to the physical layer as well as Quality of Service $(\mathrm{QoS})$ parameters. As many factors are affecting QoE, that could hide relevant information, we apply a multivariate statistical technique, called Factor Analysis (FA) [4] to reduce the whole set of measurable variables and to find out which ones have influence on the subjective video quality. This technique is also used in big data science. This could ease the design of new objective video quality metrics to estimate or predict the MOS, denoted by $\widehat{M O S}$. It must be noticed that this last step is out of the scope of this paper, because it would require more space, both to include the design as well as the performance evaluation comparing with relevant metrics.

About IEEE 802.11ac standard, it introduces enhancements to the IEEE $802.11 \mathrm{n}$, which is based on MIMO (Multiple Input Multiple Output). It contains many advanced features designed to improve the user experience, including wider radio frequency bandwidth (up to 160 MHz), more MIMO spatial streams (up to 8), Multi-User MIMO (MU-MIMO) and high-density modulation (up to 256-QAM). The standard was developed between 2011 and 2013, while devices compliant with it were released by 2015 . This $5 \mathrm{GHz}$ band has several advantages over $2.4 \mathrm{GHz}$ networks, for example they have non-overlapping channels (unlike $2.4 \mathrm{GHz}$ channels) and more channels are available for higher throughputs. Nevertheless, $5 \mathrm{GHz}$ signals suffer from greater attenuation that can be mitigated using beamforming and MIMO techniques.

The goal of this paper is to analyze the performance of IEEE 802.11ac standard under real indoor deployments from a video quality metric point of view, in particular when using Full and Ultra HD (or $4 \mathrm{~K}$ ) resolutions, while we analyze of the behavior of the measured variables 
throughout the streaming system in the WLAN to detect which ones have more influence on the video quality.

\section{RELATED WORK AND DISCUSSION}

First, we present the recent literature related to the evaluation of IEEE 802.11ac using real scenarios and equipments.

M. Dianu et al. [2] study the performance of IEEE 802.11ac in an indoor environment using UDP traffic and WPA-2 encryption. They conclude that for short distances, IEEE 802.11ac offers significantly better performance compared to IEEE $802.11 \mathrm{n}$ with data rates exceeding $700 \mathrm{Mbps}$ for a 3x3 MIMO configuration. But these improvements are very sensitive to the channel conditions with the achieved data rates rapidly declining as the distance between the transmitter and the receiver increases. Y. Zeng et al. [5] present an early performance characterization of IEEE 802.11ac standard, analyzing the impact of utilizing wider channel widths on energy efficiency and interference, that are basically the main basis for IEEE $802.11 \mathrm{ac}$. The authors show that $80 \mathrm{MHz}$ channel width yields substantial throughput improvement, but the improvements come at the cost of higher power consumption. The authors conclude that increasing the number of spatial streams is more energy efficient compared to increasing the channel width in achieving the same percentage increase in throughput. However, it is worth mentioning that the number of streams depends basically on the adapter, where most of them only support 1 or 2 spatial streams. In addition, the authors confirm that IEEE 802.11ac link suffers severe unfairness issues when it coexists with legacy IEEE 802.11 as confirmed in [2]. M. Abu-Tair et al. [3] analyze the performance and the energy efficiency of IEEE 802.11n and IEEE 802.11ac, and by comparing the results at $5 \mathrm{GHz}$, the authors find that IEEE 802.11ac achieves only 8\% more throughput than IEEE 802.11n. Finally, M. Li et al. [6] propose a QoEaware scheduling scheme for video streaming over IEEE 802.11 n/ac networks with high density of users, tunning packet delay and channel transmission rate and enhancing the video quality, measured in terms of objective Peak Signal-to-Noise Ratio (PSNR).

About video quality metrics, they are classified as Full Reference $(F R)$ and Non Reference $(N R)$ [7]. FR approaches require access both the original (or reference) and the received video, while $N R$ does not require the original video. In particular, $N R$ metrics are more interesting from the practical point of view. Using similar steps as we show in this paper, in [8] the authors design $N R$ video quality metrics based on bitstream, assisted by FR metrics, for real-time network monitoring. Notice that other $N R$ standarized metrics are O.23 (ITU-T P.1201 [9]) and G.1070 [10].

In order to design new video quality metrics we can find in the literature different techniques. Basically, they are classified in regression techniques [11] or machine learning (artificial neural networks techniques). On one hand, when we work with regression techniques, we work

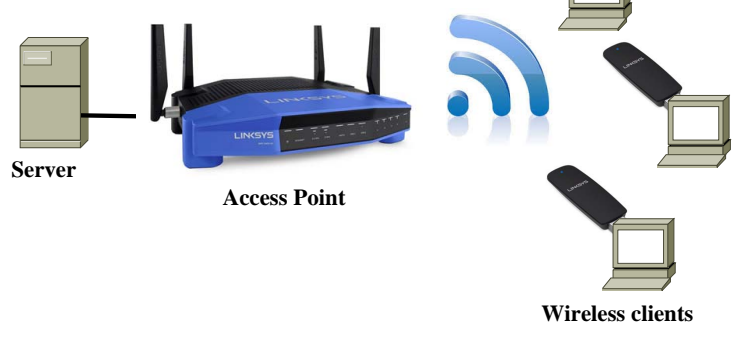

Fig. 1: Network deployment. Detail of the Video Streaming Server, the IEEE 802.11 ac/n Access Point and Wireless clients.

with all variables regardless of their inter dependences. Then with this approach, when using complex functions to fit the regression, the number of variables hamper to find out the coefficients. Thus as we suggest in this paper, FA is an interesting alternative by reducing the dimensionality of the variables, by grouping them. On the other hand, machine learning techniques and neural networks require large number of datasets, are extremely time consuming and computationally intensive [12].

It is worth mentioning that in [13] the authors use FA to model the data traffic generated by video streaming applications when using High Definition resolutions. Their goal is to define a model to support a better understanding of video stream workload characteristics and their impact on network traffic, to help in the network scheduling and resource allocation fields. Using the same technique, in [14], it shows a comprehensive simulation analysis of LTE Discontinuous Reception (DRX), allowing the Base Station (BS) to schedule User Equipments (UE) for periodic wake/sleep cycles to save energy. The authors employ FA applied to variables related directly to the DRX configuration, to determine their impact on several applications, in particular for streaming video.

We conclude from the previous works related to IEEE 802.11 ac performance evaluation, although they provide valuable information with different thorough studies, none of them have analyzed the performance in a real scenario of video streaming from a subjective point of view, that is basically the main reason for this type of standards with gigabit throughputs and by analyzing the measured variables we can help improving the design of these networks or devise new video quality metrics.

\section{EXPERIMENT DESIGN AND TEST-BED DESCRIPTION}

In order to carry out the performance analysis of the WLAN we have used the topology described in Fig. 1, where the streaming video server is connected via Gigabit Ethernet to the Access Point (AP) and the wireless clients with USB Wi-Fi adapters are located at $5 \mathrm{~m}$ distance from the AP as in a usual domestic environment. For the measurements, we used a student lab (Lab 1.1.6 at 
Computer Science Dpt. in our university) that is $20 \times 15$ square meters, with two lateral walls of glass and the other two of wood/polyester.

In our testbed, we have used off-the-shelf equipments with default configurations. The hardware description of the equipment is as follows:

1) Access Point: model Linksys WRT $1900 \mathrm{AC}$ dual band $(2.4 \& 5 \mathrm{GHz})$ Gigabit Wi-Fi router [15] (firmware 1.1.8.161917) with network standards IEEE $802.11 \mathrm{a} / \mathrm{b} / \mathrm{g} / \mathrm{n} / \mathrm{ac}$ with 4 adjustable R-SMA antennas and maximum data rates 600 and 1300 Mbps for 2.4 and $5 \mathrm{GHz}$ respectively. In particular the user manual of the AP says that it implements a draft version of the IEEE 802.11ac

2) Wireless Adapters: four USB Linksys AC 1200 wireless adapters WUSB6300 [16] (firmware 1027.5.105.2015) with 2 antennas and maximum rates 300 and $867 \mathrm{Mbps}$ for 2.4 and $5 \mathrm{GHz}$ respectively

3) Clients and server: one server connected to the access point through Gigabit Ethernet and four clients equipped with aforementioned Wi-Fi adapter. All these computers are equipped with Intel Core i7 processor, 16 GiB RAM with USB port 3.0. Clients are static on the lab's table and at the same distance from the AP.

With this configuration of antennas between the access point and receivers, MIMO is working with $4 \times 2: 2$ (maximum number of transmit antennas $\mathrm{x}$ maximum number of receive antennas : maximum number of data spatial streams). IEEE 802.11ac only operates in $5 \mathrm{GHz}$ and we will use a channel bandwidth of $80 \mathrm{MHz}$. In addition we do not use frame aggregation, allowing a fair distribution of the bandwidth as well as reducing the delay and the jitter.

The measurements have been conducted in the student labs of our university and we defined two different scenarios. It should be noted that we have different APs in the neighborhood although in different channels, as in a real environment.

In the first scenario, we measure the congestion throughput generating traffic between the server and several clients at $5 \mathrm{~m}$ from the AP. This will show us the base line of available bandwidth. In this case, each Wi-Fi client runs several simultaneous streaming connections (flows). To generate synthetic traffic we used LAN Traffic v2 (Enhanced software) [17], using UDP protocol with maximum packet sizes in IPv4 without fragmentation, 1460 and 1472 bytes respectively as in a normal video streaming scenario. To reach the saturation point of the network in order to determine the maximum throughput values, we generated packets continuously, without delay between sent packets.

In the second scenario, we analyze the performance of the WLAN with different simultaneous flows of video streaming using different wireless clients. As we expect high throughputs in these Gigabit WLAN, we used the "Big Buck Bunny" video sequence available at [18] with a duration of 1 minute with two different resolutions:

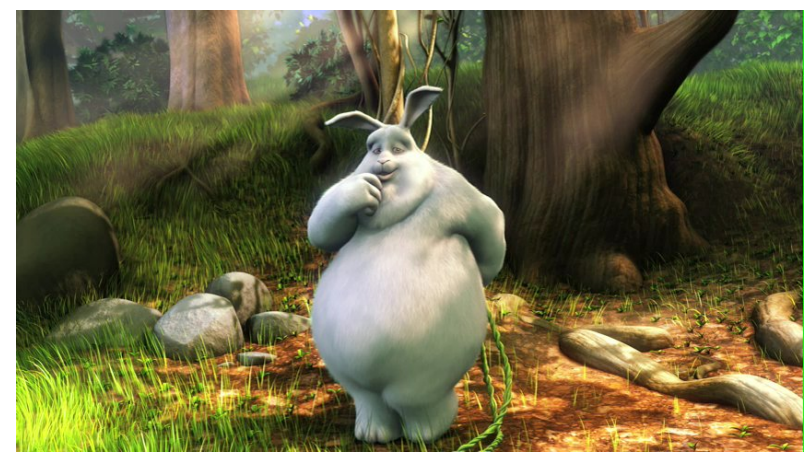

Fig. 2: A frame example from Big Buck Bunny video sequence

Full HD (HD 1920x1080) and 4K (Ultra-HD UHD, $3840 \times 2160$ ) with 30 and 60 frame per second (fps). These resolutions now on will be denoted as A, B, C and D for HD@30,HD@60,4K@30 and 4K@60 respectively. Fig. 2 shows a frame of this movie. We use the codec H.264/Advanced Video Coding (AVC) with profiles High@L4.1 for HD 30 fps, High@L4.2 for HD 60 fps and High@L5.1 for UHD-4K (both 30 and 60 fps), with a variable and adaptive GOP size. The video streaming is done using MPEG-TS (Moving Picture Experts GroupTransport Stream) as container over RTP (Real Time Protocol) and UDP. The maximum bit rates for the different streams are 16.7, 19.7, 35.1 and 37.8 Mbps respectively for A, B, C and D.

We consider the maximum number of video streams simultaneously allowed, while the subjective video quality of the streams, measured in terms of Mean Opinion Score (MOS), does not fall by more than $50 \%$ of the initial quality. That is, it will happen when start to appear permanently errors such as blocking, blurriness, freezing, etc. To asses MOS, we have used traditional subjective MOS through surveys following the recommendations given by ITU-R (BT.500-13 [19], P.910 [20]). The evaluators provide one rating for the overall video quality using a discrete five-level scale ranging from Bad (1) to Excellent (5).

The study was conducted over two sessions, each lasting less than half an hour with video sequences of $10 \mathrm{~s}$ approximately, as recommended in [19] in order to minimize evaluator fatigue. The evaluators' pool consisted of 35 under-graduate students of different ages (on average 21 years old) from our university. They are students (male and female) with a male majority, of the last course of Multimedia Engineering and they have sufficient knowledge about multimedia streaming. It should be noted that although no vision test was performed, a verbal confirmation of soundness of (corrected) vision from the subject was taken to be sufficient. For the surveys, we used the same computers described above with 22" Samsung monitor.We filtered out the measurements given by evaluators whose scores were out of a range, given by the mean and \pm two times the standard deviation. The subjective MOS has been calculated to meet a confidence interval of $95 \%$. 
For video streaming, we use the open-source FFmpeg tool [21] that allows both the configuration of the video streaming server and the client. In this case we use a buffer jitter of $500 \mathrm{~ms}$. In addition, we have used Wireshark [22], an open-source packet analyzer to capture packets with IEEE 802.11 radio information sent and measure the signal strength (in terms of Receive Signal Strength Indication (RSSI)) as well as Signal Quality (SQ) given by the adapter, Throughput (Th.), Lost Packets (LP), packet Length (Len), Delay (D) and Jitter (J). In addition to these variable, FFmpeg tool provides in their reports a muxing OverHead $(\mathrm{OH})$ included also a an input variable to be monitored.

\section{STATISTICAL MODELING: FACTOR ANALYSIS}

Factor Analysis (FA) is a multivariate statistical method used to identify the factors underlying the variables by means of grouping related variables under the same factor, where we make the assumption that an underlying causal model exists. It is a dimension reduction technique by reducing the large number of variables into few factors without sacrificing much, the power of explained variability by the variables. In other words, we can describe variability among observed, correlated variables in terms of a potentially lower number of unobserved variables called factors [4], denoted by $F_{i}$ where $i$ is the factor identifier. Variables are grouped into different factors on the basis of their interrelation. These factors partly explain the behavior of global variance of the variables. Thus, factors are unmeasured variables, defined as a linear combination of a reduced set of measurable variables, as follows:

$$
F_{i}=\text { constant }_{i}+\sum_{j=1} \alpha_{j} \cdot \text { variable }_{j}
$$

where $\alpha_{j}$ are coefficients of the linear regressions for the factors.

Variables may belong to more than one factor, but by using the factor rotation technique, these factors may be made mutually exclusive. These steps allow us to analyze the system as a linear application, where the weights of the different components are given by their eigenvalues. Thus, we set a criteria to group these variables under the same factor, when they show eigenvalues greater than one. Thus, in FA the extraction of the principal components is done by analyzing the correlation matrix of the different variables. Once we have a reduced set of components, we apply a rotation of these factors to obtain an orthogonal set by means of the Varimax algorithm and the Kaiser normalization [23].

It must be stressed that because FA is based on the correlation between the different variables, for each measurable variable we could even consider the first four standardized moments of the measured variables: mean, standard deviation, skewness and kurtosis in as similar way as shown in [13]. All of these statistics are also considered as new variables within FA. In this paper, for

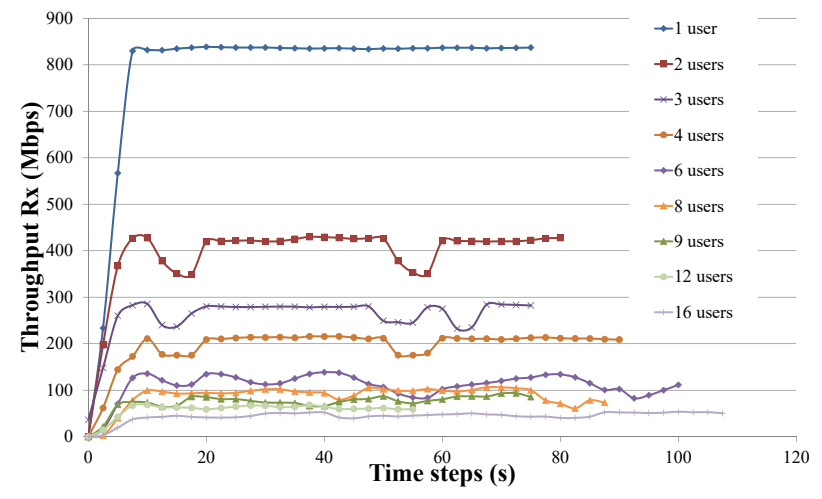

Fig. 3: Congestion Throughput in Mbps with different number of users with UDP traffic, for IEEE 802.11ac, with $80 \mathrm{MHz}$ channel bandwidth

simplicity we will consider only the first two standardized moments.

Using these factors, we could devise new video quality metrics such as $\widehat{M O S}=f\left(F_{i}\right)$, where $f()$ defines a regression function over these factors. This step is out of the scope of this paper.

\section{MONITORED VARIABLES}

With the first scenario, Fig. 3 shows the temporal variation of the throughput in the time line till it reaches the saturation point with UDP traffic. It is noteworthy that when the number of users increases, the deviation of the throughput also increases. As we can see, the available bandwidth is 840 Mbps approximately, that it is shared between the different clients.

In the second scenario and taking into account the subjective video quality, we determine the maximum number of video streams just when MOS values fall by more than $50 \%$ compared with the original MOS. Under these conditions, the maximum number of flows allowed are 20 , 20, 6 and 5 for A, B, C and D resolutions respectively. Fig. 4 shows different errors and artifacts produced in the simultaneous video streams. These artifacts produce significant subjective errors, such as blocking, blurriness, freezing, etc. It is worth mentioning that the errors in HD streams are significantly more noticeable than in UHD (4K) streams.

Now, we will analyze the behavior of these measured variables. In Table I, we describe these variables and their abbreviations. Fig. 5 shows variables related to the physical layer, that is the RSSI at the Wi-Fi adapter when receiving the video streams at different resolutions $(\mathrm{A}, \mathrm{B}$, $\mathrm{C}$ and D) and number of simultaneous clients, as well as average Signal Quality $(\%)$ given by the adapter. In order to show the RSSI fluctuations of the signal, we plot the standard deviation for each resolution on RSSI. We show the mean RSSI between different resolutions against clients as well. 


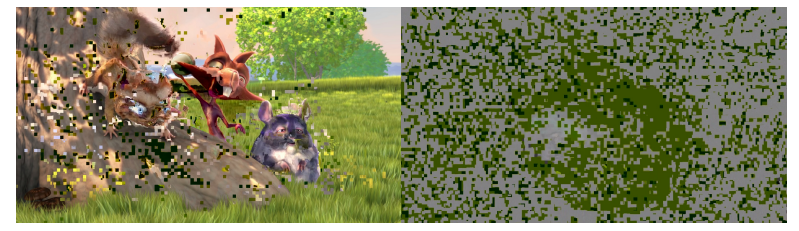

(a) A, 10 videos

(b) A, 16 videos

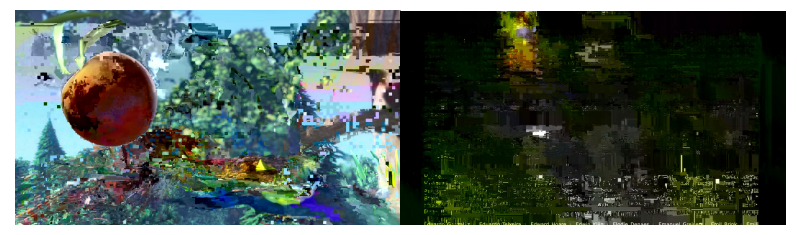

(c) B, 8 videos

(d) B, 10 videos

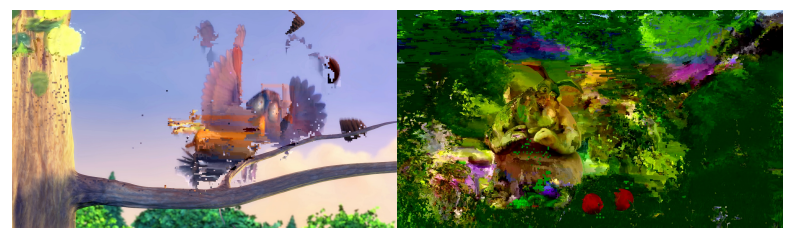

(e) C, 3 videos

(f) $\mathrm{C}, 6$ videos

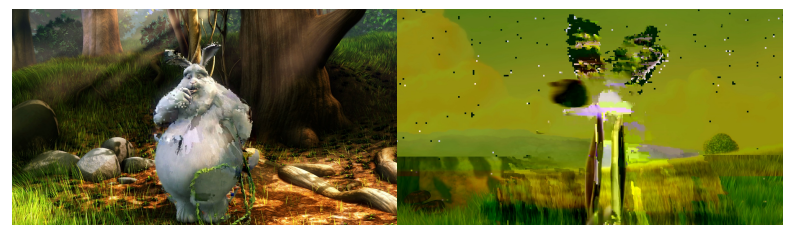

(g) D, 3 videos

(h) $\mathrm{D}, 4$ videos

Fig. 4: Received frames with several errors for different resolutions (denoted as $\mathrm{A}, \mathrm{B}, \mathrm{C}$ and $\mathrm{D}$ ), fps and number of simultaneous streaming videos

Tabla I: Measured variables and abbreviations.

\begin{tabular}{|l|c|}
\hline Variable [unit] & abbreviation \\
\hline Res [A..B] & Resolution \\
aRSSI [dBm] & avg. RSSI \\
sRSSI [dBm] & std. RSSI \\
rRRSI [dBm] & range RSSI \\
aSQ [\%] & avg. SQ \\
OH [\%] & overhead \\
LP [\%] & Lost Packets \\
mD [ms] & mean Delay \\
sD [ms] & std. Delay \\
mJ [ms] & mean Jitter \\
sJ [ms] & std. Jitter \\
mT [Mbps] & mean Throughput \\
sT [Mbps] & std. Throughput \\
mLen [byte] & mean Length Packet \\
sLen [byte] & std. Length Packet \\
\hline
\end{tabular}

Fig. 6 shows all the measured variables related to QoS, such as Jitter (ms), Delay (ms), Th. (Mbps) and Packet Length (bytes) both with mean and standard deviation, as well as Lost Packets (\%), for different video resolutions (A, B, C and D) against clients. At a first glance, it can be seen from this figure, that values remain constant except for Jitter. This is due to many things. Both clients and servers are on the same LAN thus the time uncertainty is reduced as well as the MAC algorithms split or share the available bandwidth in a fair way because of the high throughput. However, these arguments do not go with Jitter, that clearly increases as the number of clients increase, basically as a result of the traffic as well as the internal queue management in the AP.

In addition, we measure MOS values as shown in Fig. 7. It is important to highlight that we see a video quality improvement when increasing fps. Besides, subjective errors due to blocking, blurriness, freezing, etc., that highly impact the subjective video quality, appear randomly independently of the resolution.

As a preliminary step, in Fig. 8 we show the correlation coefficients for the different variables against MOS. By analyzing the significance level of this correlation coefficients, we see that $a R S S I, s R S S I, r R R S I, a S Q, m D$ and $m J$ are not correlated with MOS.

\section{STATISTICAL ANALYSIS AND RESULTS}

We have used SPSS.r22 software package for statistical analysis and Matlab R2015a. Following the steps explained in Section IV, to perform a FA, first we have to analyze the correlation matrix, shown in Table II, on the basis of which variables are grouped into factors. Based on this information, we determine the number factors and map the variables into them, taking into account the variance explained of the data. In this case, we find that with 4 factors we explain $89.46 \%$ of the total variance. Each factor explains 49.93, 19.13, 11.48 and $8.93 \%$ respectively. The results of this mapping process are shown in Table III, where each variable is assigned (after performing a Varimax rotation), based on the Pearson's coefficient $\left(R^{2}\right)$, to each factor. At this point from our results and for this scenario, on one hand it worth mentioning that some factors are less relevant than others, in particular $F_{3}$ and $F_{4}$ that only explain 11.48 and $8.93 \%$ respectively of the total variance. These factors include variables related to the physical layer (RSSI and SQ). On the other hand, on $F_{1}$ we see the main critical variables.

Once we have found out the factors composition, using linear regression we define the expressions that determine them, as shown in Table IV along with their $R^{2}$ coefficients. Notice that $O H, s L e n, m J$ from $F_{1}$ and $m T$ from $F_{2}$ have been excluded when modeling these factors due to their low contribution.

Now, with these factors (a reduced data set) we could perform different regression techniques in order to devise a $N R$ new video quality and would assist the design of new $N R$ video quality metrics, but this is out of the scope of this paper. Besides, it must be stressed that the presented data is biased version because we did not include all data. We skipped data related to bad MOS measurements because we focused only on the performance evaluation of IEEE 802.11ac.

\section{CONCLUSION}

In this paper we have studied the measurement-based performance of IEEE 802.11ac standard, both analyzing the maximum saturation throughput and the maximum number of high definition streaming video flows under a subjective video quality point of view. From these results, 


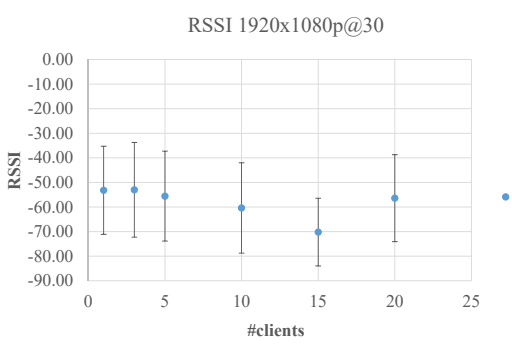

(a)

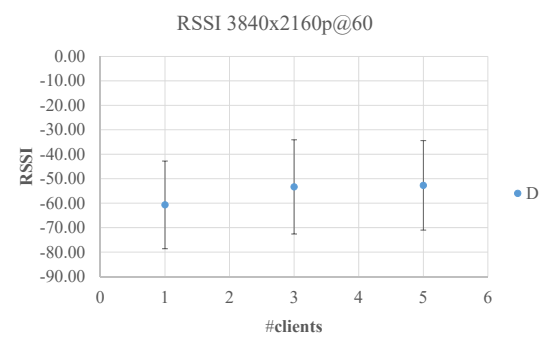

(d)

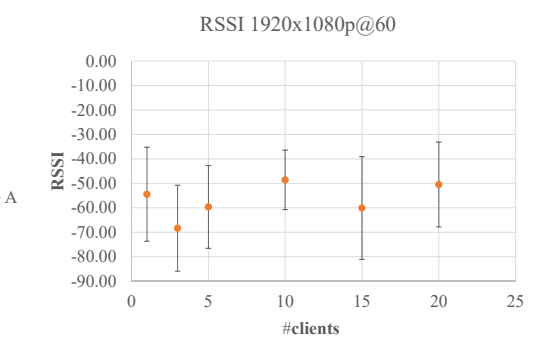

(b)

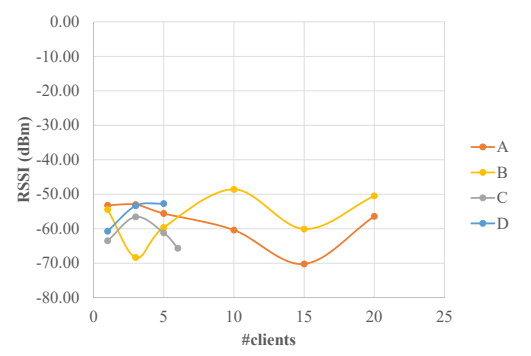

(e)

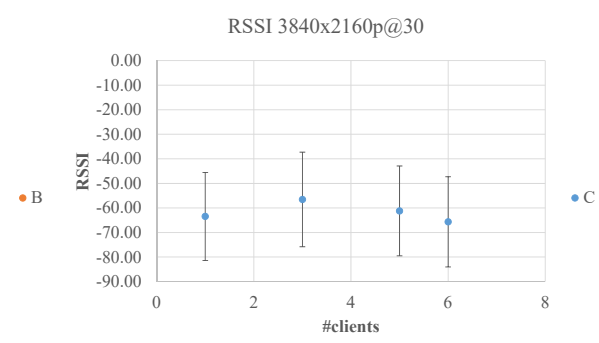

(c)

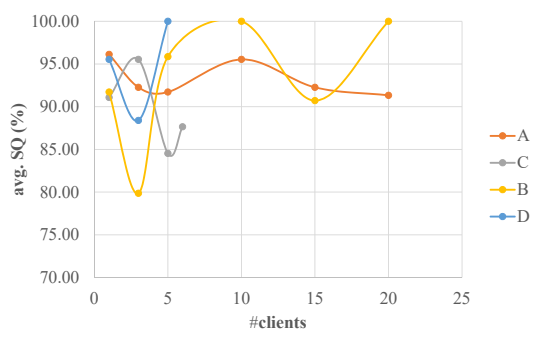

(f)

Fig. 5: RSSI (dBm) with detail of standard deviation and average Signal Quality (\%) for the different video resolutions (denoted as A, B, C and D) against clients

Tabla II: Correlation matrix for the different measured variables.

\begin{tabular}{|c|c|c|c|c|c|c|c|c|c|c|c|c|c|c|c|}
\hline & 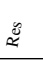 & & 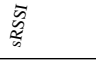 & $\stackrel{\mathscr{Z}}{\Xi}$ & : & T & 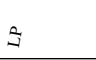 & हิ & $\therefore$ & $\grave{\Xi}$ & 5 & $\cong$ & 5 & छี & 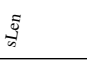 \\
\hline Res & 1 & 0.01 & 0.19 & -0.18 & -0.03 & -0.52 & -0.95 & -0.75 & -0.27 & -0.41 & -0.46 & 0.98 & 0.98 & 0.49 & -0.55 \\
\hline aRSSI & & 1 & -0.07 & 0.01 & 0.62 & 0.20 & 0.10 & -0.25 & 0.09 & 0.31 & 0.07 & -0.06 & -0.06 & -0.23 & 0.25 \\
\hline sRSSI & & & 1 & 0.72 & -0.46 & -0.33 & -0.29 & 0.10 & -0.17 & -0.16 & -0.21 & 0.26 & 0.26 & 0.34 & -0.37 \\
\hline rRRSI & & & & 1 & -0.31 & -0.24 & 0.07 & 0.43 & -0.16 & -0.22 & -0.10 & -0.08 & -0.08 & 0.28 & -0.29 \\
\hline aSQ & & & & & 1 & 0.14 & 0.12 & -0.20 & 0.03 & 0.27 & 0.04 & -0.09 & -0.09 & -0.18 & 0.22 \\
\hline $\mathrm{OH}$ & & & & & & 1 & 0.65 & 0.11 & 0.86 & 0.60 & 0.90 & -0.65 & -0.66 & -0.99 & 0.98 \\
\hline LP & & & & & & & 1 & 0.61 & 0.36 & 0.46 & 0.54 & -0.97 & -0.97 & -0.63 & 0.69 \\
\hline $\mathrm{mD}$ & & & & & & & & 1 & 0.17 & 0.19 & 0.33 & -0.61 & -0.61 & -0.06 & 0.04 \\
\hline sD & & & & & & & & & 1 & 0.54 & 0.98 & -0.35 & -0.36 & -0.85 & 0.76 \\
\hline $\mathrm{mJ}$ & & & & & & & & & & 1 & 0.57 & -0.46 & -0.47 & -0.59 & 0.57 \\
\hline $\mathrm{sJ}$ & & & & & & & & & & & 1 & -0.53 & -0.53 & -0.88 & 0.81 \\
\hline $\mathrm{mT}$ & & & & & & & & & & & & 1 & 1 & 0.63 & -0.69 \\
\hline sT & & & & & & & & & & & & & 1 & 0.63 & -0.70 \\
\hline mLen & & & & & & & & & & & & & & 1 & -0.99 \\
\hline sLen & & & & & & & & & & & & & & & 1 \\
\hline
\end{tabular}

we see that this standard fails to match the expectations created. It must be pointed out that these results heavily depend on the implementation of the IEEE 802.11ac standard of the commercial access points. These comments agree with [2], [3] because the network performance results in real environments are quite disparate from those we should expect from the standard. Nevertheless, although commercial products are not still 100\% IEEE 802.11ac compliant, they actually can support several simultaneous UHD (4K) video streams at home (in our case, till 3 with an excellent quality).

Finally, we see how using FA we define a reduced data set, useful in particular when managing many variables and we want to devise a new $N R$ video metric. This technique is used in big data science. In addition, we have seen that the measured variables related to the physical layer, are not relevant in the design of new $N R$ video quality metrics at least if we reach a certain threshold of RSSI.

\section{ACKNOWLEDGMENT}

This work was supported by the Universitat de València under the projects UV-INV-PRECOMP14-207134, UVINVAE15-339582, by the Generalitat Valenciana under the 


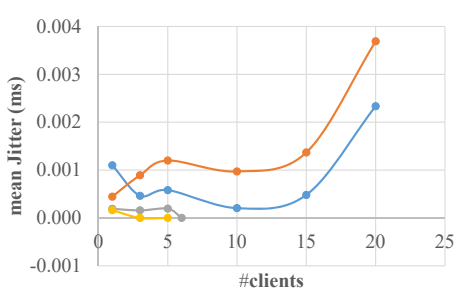

(a) Mean jitter (ms)

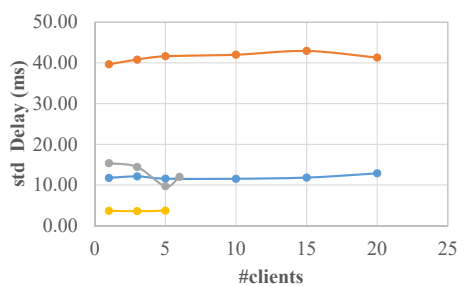

(d) Std. delay (ms)

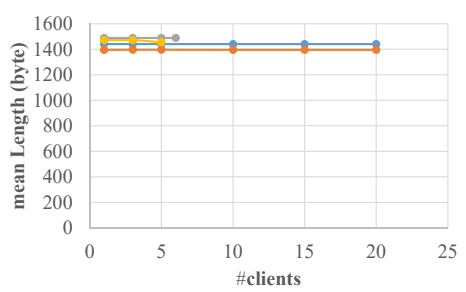

(g) Mean p.length (byte)
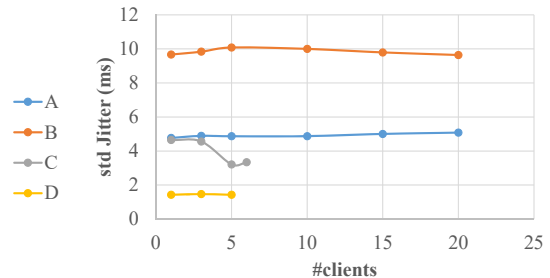

(b) Std. jitter (ms)

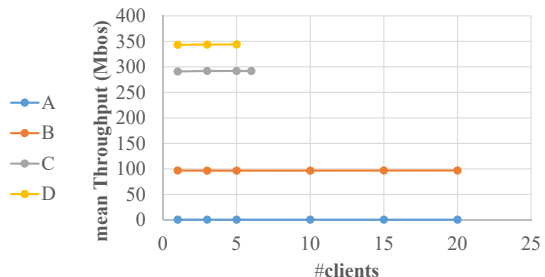

(e) Mean Th. (Mbps)

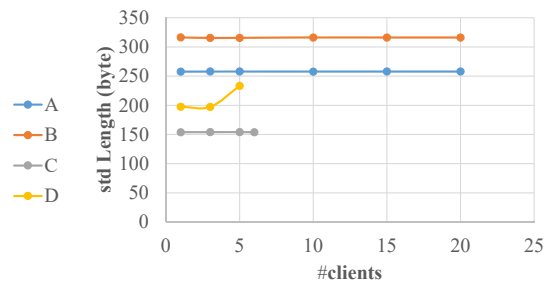

(h) Std. p.length (byte)

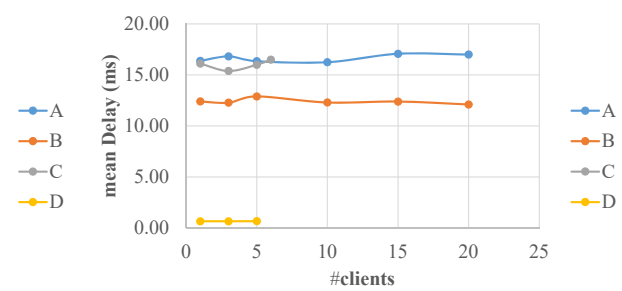

(c) Mean delay (ms)

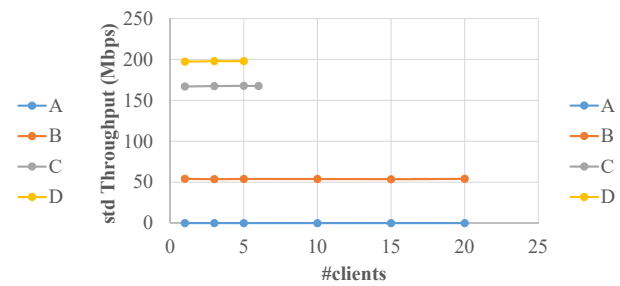

(f) Std. Th. (Mbps)

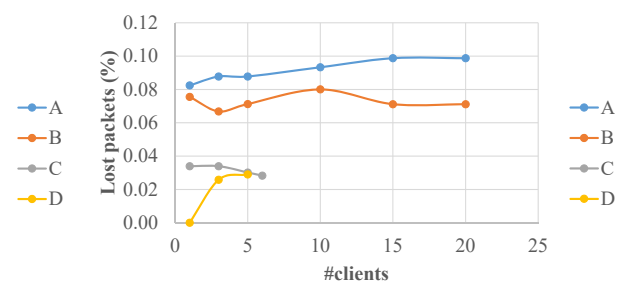

(i) Lost packets (\%)

Fig. 6: Relevant measured variables jitter (ms), delay (ms), throughput (Th.) (Mbps), packet length (bytes) with mean and standard deviation as well as lost packets $(\%)$, for different video resolutions against clients

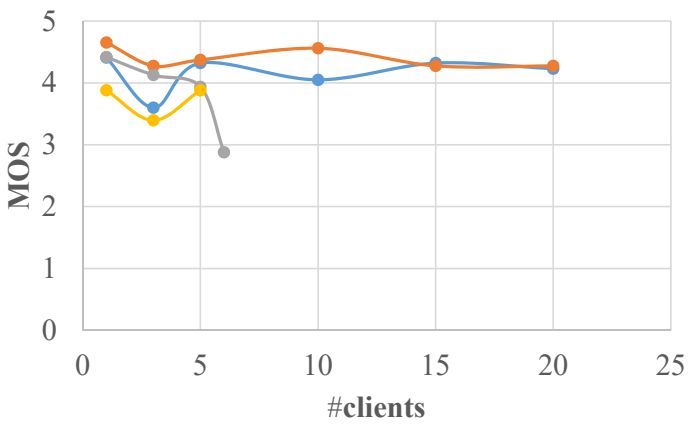

Fig. 7: MOS values for the different video resolutions (denoted as A, B, C and D) against clients

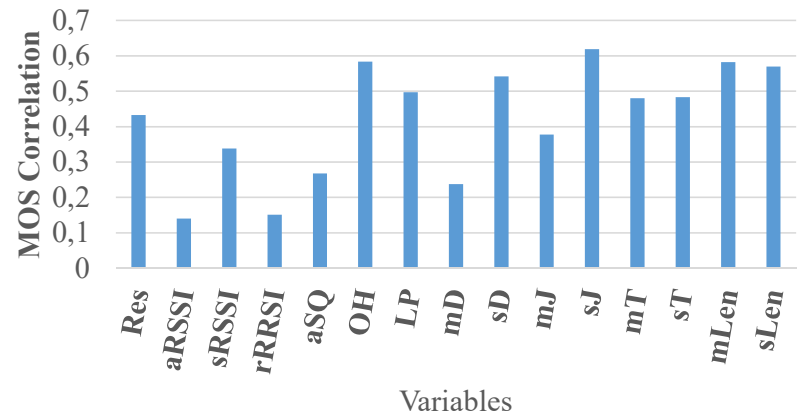

Fig. 8: Correlation coefficients of the measured variables with MOS

\section{REFERENCIAS}

project GV-2016-002 and by the Ministry of Economy under the project BIA2016-76957-C3-1-R.

[1] Cisco, "Visual Networking Index Global Mobile Data Traffic Forecast, 20172020. White paper." Cisco Systems, Corp., Tech. Rep., 02 2017. [Online]. Available: http://www.cisco.com/c/en/us/ 
Tabla III: Rotated component matrix with their loading factor

\begin{tabular}{|l|c|c|c|c|}
\hline Variable & $F_{1}$ & $F_{2}$ & $F_{3}$ & $F_{4}$ \\
\hline sD & $\mathbf{0 . 9 6}$ & -0.05 & 0.00 & -0.06 \\
sJ & $\mathbf{0 . 9 3}$ & -0.26 & 0.00 & -0.06 \\
OH & $\mathbf{0 . 9 2}$ & -0.31 & -0.18 & 0.09 \\
mLen & $\mathbf{- 0 . 9 1}$ & 0.28 & 0.21 & -0.13 \\
sLen & $\mathbf{0 . 8 4}$ & -0.36 & -0.25 & 0.18 \\
mJ & $\mathbf{0 . 5 9}$ & -0.27 & -0.05 & 0.33 \\
Res & -0.24 & $\mathbf{0 . 9 7}$ & -0.01 & -0.01 \\
mT & -0.36 & $\mathbf{0 . 9 2}$ & 0.08 & -0.07 \\
sT & -0.37 & $\mathbf{0 . 9 1}$ & 0.08 & -0.07 \\
LP & 0.36 & $\mathbf{- 0 . 9 0}$ & -0.09 & 0.10 \\
mD & 0.00 & $\mathbf{- 0 . 7 8}$ & 0.30 & -0.26 \\
sRSSI & -0.12 & 0.18 & $\mathbf{0 . 9 1}$ & -0.13 \\
rRRSI & -0.19 & -0.24 & $\mathbf{0 . 8 9}$ & -0.03 \\
aRSSI & 0.15 & 0.06 & 0.11 & $\mathbf{0 . 9 3}$ \\
aSQ & 0.01 & -0.03 & -0.37 & $\mathbf{0 . 8 2}$ \\
\hline
\end{tabular}

Tabla IV: Expressions of the factors

$$
\begin{array}{ll}
F_{1}= & 18.713+0.084 \cdot s D-0.013 \cdot m L e n-0.236 \cdot s J ;\left(R^{2}=\right. \\
& 0.974) \\
F_{2}= & -5.304+3.282 \cdot R e s-0.031 \cdot s T+0.09 \cdot m D-7.917 . \\
& L P ;\left(R^{2}=0.993\right) \\
F_{3}= & -10.057+0.252 \cdot s R S S I+0.114 \cdot r R R S I ;\left(R^{2}=0.939\right) \\
F_{4}= & -0.424+0.114 \cdot a R S S I+0.076 \cdot a S Q ;\left(R^{2}=0.956\right)
\end{array}
$$

solutions/collateral/service-provider/visual-networking-index-vni/ mobile-white-paper-c11-520862.html

[2] M.-D. Dianu, J. Riihijarvi, and M. Petrova, "Measurement-based study of the performance of IEEE 802.11ac in an indoor environment," in Communications (ICC), 2014 IEEE International Conference on, June 2014, pp. 5771-5776.

[3] M. Abu-Tair and S. Bhatti, "Upgrading 802.11 deployments: A Critical Examination of Performance," in Advanced Information Networking and Applications (AINA), 2015 IEEE 29th International Conference on, March 2015, pp. 844-851.

[4] R. L. Gorsuch, Factor analysis. Hillsdale: Lawrence Erlbaum Associates, 1983.

[5] Y. Zeng, P. Pathak, and P. Mohapatra, "A first look at 802.11ac in action: Energy efficiency and interference characterization," in Networking Conference, 2014 IFIP, June 2014, pp. 1-9.

[6] M. Li, P. H. Tan, S. Sun, and Y. H. Chew, "Qoe-aware scheduling for video streaming in $802.11 \mathrm{n} /$ ac-based high user density networks," in 2016 IEEE 83rd Vehicular Technology Conference (VTC Spring), May 2016, pp. 1-5.

[7] S. Chikkerur, V. Sundaram, M. Reisslein, and L. Karam, "Objective Video Quality Assessment Methods: A Classification, Review, and Performance Comparison," Broadcasting, IEEE Transactions on, vol. 57 , no. 2, pp. 165-182, June 2011.

[8] I. Sedano, K. Brunnström, M. Kihl, and A. Aurelius, "Fullreference video quality metric assisted the development of no-reference bitstream video quality metrics for real-time network monitoring," EURASIP Journal on Image and Video Processing, vol. 2014, no. 1, pp. 1-15, 2014. [Online]. Available: http://dx.doi.org/10.1186/1687-5281-2014-4

[9] ITU-T, "Parametric non-intrusive assessment of audiovisual media streaming quality," P.1201 Amendment 2, vol. Series P: Terminals and subjective and objective assessment methods, 12 2013. [Online]. Available: http://www.itu.int/rec/T-REC-P. 1201-201312-I!Amd2

[10] —-, "Opinion Model for Video-Telephony Applications." G.1070, 2012a, vol. Series G: Transmission systems and media, digital systems and networks, 7 2012. [Online]. Available: https://www.itu.int/rec/T-REC-G.1070-201207-I/en

[11] M. Alreshoodi and J. Woods, "Survey on qoe $\backslash$ qos correlation models for multimedia services," International Journal of Distributed and Parallel Systems (IJDPS), vol. 4, 2013. [Online]. Available: http://arxiv.org/abs/1306.0221

[12] S. Aroussi and A. Mellouk, "Survey on machine learning-based qoe-qos correlation models," in 2014 International Conference on Computing, Management and Telecommunications (ComManTel), April 2014, pp. 200-204.

[13] A. K. A. Tamimi, R. Jain, and C. So-In, "Statistical analysis and modeling of high definition video traces," in ICME. IEEE, 2010, pp. 596-601.

[14] G. Stea and A. Virdis, "A comprehensive simulation analysis of LTE Discontinuous Reception (DRX)," Computer Networks, vol. 73, no. 0, pp. $22-40,2014$.

[15] "Linksys WRT 1900 AC dual band," 2015, accessed: 1/10/2015 [Online]. Available: http://www.linksys.com/us/p/P-WRT1900AC

[16] "Linksys USB AC1200 wireless adapters WUSB6300," 2015, accessed: 1/10/2015. [Online]. Available: http://www.linksys.com/ us/p/P-WUSB6300/

[17] "LAN Traffic v2 (Enhanced software)," 2015, accessed: 8/2/2015. [Online]. Available: http://www.zti-communications. com/documentation/LanTrafficV2Enhanced_UserGuide.pdf

[18] "Big Buck Bunny, Sunflower version." 2013, accessed: 15/9/2015 [Online]. Available: http://bbb3d.renderfarming.net/download.html

[19] ITU-R, "Methodology for the Subjective Assessment of the Quality of Television Pictures," BT.500-13, 1 2013. [Online]. Available: https://www.itu.int/dms_pubrec/itu-r/rec/bt/R-REC-BT. 500-13-201201-I!!PDF-E.pdf

[20] _ - "Subjective Video Quality Assessment Methods for Multimedia Applications," P.910, 91999.

[21] FFmpeg, "Tools," 2016. [Online]. Available: https://www.ffmpeg. org/

[22] A. Orebaugh, G. Ramirez, J. Burke, and L. Pesce, Wireshark \& Ethereal Network Protocol Analyzer Toolkit (Jay Beale's Open Source Security). Syngress Publishing, 2006.

[23] H. T. Kaiser, "The Application of Electronic Computers to factor analysis," Educational and Psychological Measurement, vol. 20, p. $141151,1960$. 